ESAIM: COCV 21 (2015) 359-371

DOI: $10.1051 / \mathrm{cocv} / 2014016$
ESAIM: Control, Optimisation and Calculus of Variations

www.esaim-cocv.org

\title{
SYMMETRY BREAKING IN A CONSTRAINED CHEEGER TYPE ISOPERIMETRIC INEQUALITY
}

\author{
Barbara Brandolini ${ }^{1}$, Francesco Della Pietra ${ }^{1}$, Carlo Nitsch $^{1}$ \\ And Cristina Trombetti ${ }^{1}$
}

\begin{abstract}
The study of the optimal constant $\mathcal{K}_{q}(\Omega)$ in the Sobolev inequality

$$
\|u\|_{L^{q}(\Omega)} \leq \frac{1}{\mathcal{K}_{q}(\Omega)}\|D u\|\left(\mathbb{R}^{n}\right), \quad 1 \leq q<1^{*},
$$

for BV functions which are zero outside $\Omega$ and with zero mean value inside $\Omega$, leads to the definition of a Cheeger type constant. We are interested in finding the best possible embedding constant in terms of the measure of $\Omega$ alone. We set up an optimal shape problem and we completely characterize, on varying the exponent $q$, the behaviour of optimal domains. Among other things we establish the existence of a threshold value $1 \leq \tilde{q}<1^{*}$ above which the symmetry of optimal domains is broken. Several differences between the cases $n=2$ and $n \geq 3$ are emphasized.
\end{abstract}

Mathematics Subject Classification. 49Q20, 39B05.

Received November 7, 2013. Revised February 21, 2014.

Published online October 10, 2014.

\section{Statement of the Problem and main Results}

Let $\Omega$ be a bounded, open set of $\mathbb{R}^{n}, n \geq 2$, and let $1 \leq q<1^{*}=\frac{n}{n-1}$. Denoting by $B V_{0}(\Omega)=$ $\left\{u \in B V\left(\mathbb{R}^{n}\right): u \equiv 0\right.$ in $\left.\mathbb{R}^{n} \backslash \Omega\right\}$ it is well-known that there exists a constant $\mathcal{C}_{q}^{n}(\Omega)$ such that

$$
\|u\|_{L^{q}(\Omega)} \leq \frac{1}{\mathcal{C}_{q}(\Omega)}\|D u\|\left(\mathbb{R}^{n}\right)
$$

for all $u \in B V_{0}(\Omega)$. Here $\|D u\|\left(\mathbb{R}^{n}\right)$ denotes the total variation of $u$ in $\mathbb{R}^{n}$.

The least possible constant such that (1.1) holds true is given by

$$
\mathcal{C}_{q}(\Omega)=\min \left\{\frac{\|D u\|\left(\mathbb{R}^{n}\right)}{\|u\|_{L^{q}(\Omega)}}: u \in B V_{0}(\Omega), u \neq \equiv 0\right\}
$$

\footnotetext{
Keywords and phrases. Cheeger inequality, optimal shape, symmetry and asymmetry.
}

1 Università degli Studi di Napoli "Federico II", Dipartimento di Matematica e Applicazioni "R. Caccioppoli", Complesso Monte S. Angelo - Via Cintia, 80126 Napoli, Italia. brandolini@unina.it; f.dellapietra@unina.it; c.nitsch@unina.it; cristina@unina.it 
a quantity that is well-known in literature since it coincides with the so-called Cheeger constant [5] (see also the survey paper [19] and the references therein):

$$
\mathcal{C}_{q}(\Omega)=\min \left\{\frac{P(E)}{|E|^{\frac{1}{q}}}: E \subseteq \Omega,|E|>0\right\} .
$$

Here by $P(E)$ and $|E|$ we denote the perimeter and the Lebesgue measure of $E$ respectively.

An elementary scaling argument enforces $\mathcal{C}_{q}(\Omega)|\Omega|^{\frac{1}{q}-\frac{n-1}{n}}$ to be invariant under dilations, therefore it is possible to optimize such a product over all bounded, open sets $\Omega$. Indeed an interesting consequence of the isoperimetric inequality is that

$$
\mathcal{C}_{q}(\Omega)|\Omega|^{\frac{1}{q}-\frac{n-1}{n}} \geq n \omega_{n}^{\frac{1}{n}}
$$

where $\omega_{n}$ denotes the measure of the unit ball in $\mathbb{R}^{n}$. Hence in the class of bounded, open sets of given measure balls minimize $\mathcal{C}_{q}(\Omega)$. Furthermore, given any bounded, open set $\Omega$, it holds that

$$
\|u\|_{L^{q}(\Omega)} \leq \frac{|\Omega|^{\frac{1}{q}-\frac{n-1}{n}}}{n \omega_{n}^{\frac{1}{n}}}\|D u\|\left(\mathbb{R}^{n}\right)
$$

for all $u \in B V_{0}(\Omega)$.

The study of optimal constants in Sobolev-Poincaré inequalities for BV functions has been very popular since several decades. Many results can be found for instance in [16], and more recently in [6,10-12].

In this paper we consider the following minimization problem

$$
\mathcal{K}_{q}(\Omega)=\min \left\{\frac{\|D u\|\left(\mathbb{R}^{n}\right)}{\|u\|_{L^{q}(\Omega)}}: u \in B V_{0}(\Omega), u \not \equiv 0, \int_{\Omega} u \mathrm{~d} x=0\right\},
$$

carrying the Sobolev inequality

$$
\|u\|_{L^{q}(\Omega)} \leq \frac{1}{\mathcal{K}_{q}(\Omega)}\|D u\|\left(\mathbb{R}^{n}\right)
$$

holding for functions $u \in B V_{0}(\Omega)$ having zero mean value. Since in general $\mathcal{C}_{q}(\Omega) \leq \mathcal{K}_{q}(\Omega)$, in comparison with (1.1), we are trading the restriction to zero mean value functions for a better embedding constant. Even in this case, scaling arguments enforce $\mathcal{K}_{q}(\Omega)|\Omega|^{\frac{1}{q}-\frac{n-1}{n}}$ to be invariant under dilations and the present paper is devoted to the study of the optimal lower bound in the wake of (1.2) and to a complete characterization of the optimal sets (from now on called "minimizers") on which $\mathcal{K}_{q}(\Omega)|\Omega|^{\frac{1}{q}-\frac{n-1}{n}}$ achieves the lower bound.

To this aim we rewrite $\mathcal{K}_{q}(\Omega)$ in terms of geometric quantities, such as perimeters and measures of subsets of $\Omega$. For a given bounded open set $\Omega$ we define

$$
\Phi(\Omega)=\left\{\left(E_{1}, E_{2}\right): E_{1}, E_{2} \subseteq \Omega, E_{1} \cap E_{2}=\emptyset,\left|E_{1}\right|>0,\left|E_{2}\right|>0\right\},
$$

and prove the following.

Theorem 1.1. If $\Omega$ is a bounded open set of $\mathbb{R}^{n}, n \geq 2$, and $1 \leq q<1^{*}$, then

$$
\mathcal{K}_{q}(\Omega)=\min \left\{\frac{\frac{P\left(E_{1}\right)}{\left|E_{1}\right|}+\frac{P\left(E_{2}\right)}{\left|E_{2}\right|}}{\left(\left|E_{1}\right|^{1-q}+\left|E_{2}\right|^{1-q}\right)^{\frac{1}{q}}}:\left(E_{1}, E_{2}\right) \in \Phi(\Omega)\right\} .
$$

Taking advantage of (1.5), we are able to characterize the minimizers. Astonishingly, the minimization is very sensitive to the choice of $n$ and $q$. A symmetry breaking phenomenon appears above a threshold value of the exponent $q$. Furthermore for certain choices of $n$ and $q$ minimizers are not even unique. Our main result is the following. 
Theorem 1.2. For all $n \geq 2$ every minimizer of $\mathcal{K}_{q}(\Omega)$ in the class of bounded, open sets with given measure, is union of two disjoint balls. Shape and uniqueness of such minimizers depends on $n$ and $q$. More specifically, there exists $\tilde{q}=\tilde{q}(n) \in] 1,1^{*}[$ such that, when $q<\tilde{q}$, the minimizer is unique and the two balls composing the minimizer have the same radius, while, when $q>\tilde{q}$, the minimizer is unique and the two balls composing the minimizer have different radii. Moreover:

(1) If $n=2$, then $\tilde{q}=\frac{7}{4}$, the minimizer is unique even at $q=\tilde{q}$ and consists in the union of two disjoint balls with equal radii.

(2) For all $n \geq 3$, the minimizer is not unique at $q=\tilde{q}$, indeed there are exactly two minimizers one of which is the union of two disjoint balls with equal radii.

Remark 1.3. We point out some additional properties that will be deduced during the proof of the Theorem 1.2. Assume that we work with the class of bounded, open sets of given measure. When $n=2$, the radii of the balls composing the unique minimizer change continuously for $q \in[1,2$ [ and the largest one is nondecreasing with respect to $q$. The case $n \geq 3$ shows some differences. Bearing in mind that the exact value $\tilde{q}$ is not explicitly given, we can bound it from above and below by $1+\frac{1}{n}+\frac{1}{n^{2}}$ and $1+\frac{1}{n}$ respectively. More important, crossing the threshold value $\tilde{q}$ the minimizer abruptly jumps from two balls of equal radii to two balls of unequal radii. For $q=\tilde{q}$ minimizing pairs of balls of equal and unequal radii coexist. Considering the minimizing pairs of unequal radii, for $q \geq \tilde{q}$, the radius of the largest ball continuously increases with respect to $q$.

Finally, regardless the value $n \geq 2$ the minimizing pair always degenerates to one ball as $q \rightarrow 1^{*}$.

A different point of view to look at our problem consists in considering the minimization in (1.3) as relaxed form of

$$
\mathcal{K}_{q}(\Omega)=\inf \left\{\frac{\int_{\Omega}|\nabla u| \mathrm{d} x}{\left(\int_{\Omega}|u|^{q} \mathrm{~d} x\right)^{\frac{1}{q}}}: u \in W_{0}^{1,1}(\Omega), u \neq \equiv \text { and } \int_{\Omega} u \mathrm{~d} x=0\right\} .
$$

In this case we can address to $\mathcal{K}_{q}(\Omega)$ as the "twisted eigenvalue" of the 1-Laplacian or "twisted Cheeger constant", that means $\mathcal{K}_{q}(\Omega)$ is the $B V$ counterpart of the so-called twisted eigenvalue for the Laplacian

$$
\lambda^{T}(\Omega)=\min \left\{\frac{\int_{\Omega}|\nabla u|^{2} \mathrm{~d} x}{\int_{\Omega}|u|^{2} \mathrm{~d} x}: u \in H_{0}^{1}(\Omega), u \neq \equiv \text { and } \int_{\Omega} u \mathrm{~d} x=0\right\} .
$$

To our knowledge the term "twisted eigenvalue" was first introduced by Barbosa and Bérard in [1]. Later Freitas and Henrot in [13] employed symmetrization arguments to show that the pairs of disjoint balls of equal radii are the unique minimizers of $\lambda^{T}(\Omega)$ among all bounded, open sets of given measure. For the interested reader, generalization of the twisted Laplacian eigenvalue in different directions have already been studied for instance in $[2,3,8,18]$.

In the spirit of [13], one might expect that also minimizers for $\mathcal{K}_{q}(\Omega)$ are pairs of disjoint balls of equal radii, and Theorem 1.2 contradicts this intuition.

The picture that we get is much more similar to the one obtained for certain 1-dimensional Wirtinger inequalities in $[4,7,9,14,17]$ where the occurrence of symmetric and asymmetric minimizers have been completely settled. In dimension greater than 1, symmetry breaking for $p$-Laplacian twisted eigenvalue problems, for certain range of exponents, have been observed in an interesting remark by Nazarov recently appeared in [18].

We finally thank the referee for having pointed out the following estimate

$$
\mathcal{K}_{1}(\Omega) \leq \mathcal{C}_{1,2}(\Omega)
$$


where

$$
\mathcal{C}_{1,2}=\min _{\left(E_{1}, E_{2}\right) \in \Phi(\Omega)} \max \left\{\mathcal{C}_{1}\left(E_{1}\right), \mathcal{C}_{1}\left(E_{2}\right)\right\}
$$

is the second Cheeger constant introduced in [20]. If we generalise this notion, and define

$$
\mathcal{C}_{q, 2}=\min _{\left(E_{1}, E_{2}\right) \in \Phi(\Omega)} \max \left\{\mathcal{C}_{q}\left(E_{1}\right), \mathcal{C}_{q}\left(E_{2}\right)\right\}, \quad q \in\left[1,1^{*}[,\right.
$$

by using the concavity of the function $f(t)=t^{\frac{1}{q}}$, we get

$$
\mathcal{K}_{q}(\Omega) \leq 2^{1-\frac{1}{q}} \mathcal{C}_{q, 2}(\Omega) .
$$

\section{Proof of the Theorem 1.1: Reduction to Characteristic functions}

The main idea is to show that it is possible to study problem (1.3) by considering only test functions whose positive and negative parts are characteristic functions up to multiplicative factors.

Let us introduce the following notation. For any $u \in B V_{0}(\Omega)$ we set

$$
F_{q}(u)=\frac{\|D u\|\left(\mathbb{R}^{n}\right)}{\|u\|_{L^{q}(\Omega)}} .
$$

Clearly, if as usual $\chi_{E}$ denotes the characteristic function of a set $E \subseteq \mathbb{R}^{n}$, recalling (1.4),

$$
F_{q}\left(\left|E_{2}\right| \chi_{E_{1}}-\left|E_{1}\right| \chi_{E_{2}}\right)=\frac{\frac{P\left(E_{1}\right)}{\left|E_{1}\right|}+\frac{P\left(E_{2}\right)}{\left|E_{2}\right|}}{\left(\left|E_{1}\right|^{1-q}+\left|E_{2}\right|^{1-q}\right)^{\frac{1}{q}}}, \quad\left(E_{1}, E_{2}\right) \in \Phi(\Omega) .
$$

We denote

$$
Q\left(E_{1}, E_{2}\right)=\frac{\frac{P\left(E_{1}\right)}{\left|E_{1}\right|}+\frac{P\left(E_{2}\right)}{\left|E_{2}\right|}}{\left(\left|E_{1}\right|^{1-q}+\left|E_{2}\right|^{1-q}\right)^{\frac{1}{q}}}
$$

and we define

$$
\ell_{q}(\Omega)=\inf _{\left(E_{1}, E_{2}\right) \in \Phi(\Omega)} Q\left(E_{1}, E_{2}\right) .
$$

First we prove that the infimum in (2.1) is attained. The classical isoperimetric inequality implies that

$$
Q\left(E_{1}, E_{2}\right) \geq n \omega_{n}^{\frac{1}{n}} \frac{\left|E_{1}\right|^{-\frac{1}{n}}+\left|E_{2}\right|^{-\frac{1}{n}}}{\left(\left|E_{1}\right|^{1-q}+\left|E_{2}\right|^{1-q}\right)^{\frac{1}{q}}}
$$

and, being $1 \leq q<1^{*}$, the right-hand side diverges as $\left(\left|E_{1}\right|,\left|E_{2}\right|\right) \rightarrow(0,0)$. Hence, if $\left(E_{1}^{k}, E_{2}^{k}\right)$ is a sequence of couples of domains in $\Phi(\Omega)$ which minimizes $(2.1)$ as $k \rightarrow+\infty$, the sequences $\chi_{E_{1}^{k}}, \chi_{E_{2}^{k}}$ are bounded in $B V$ and, up to subsequences, strongly converge in $L^{1}(\Omega)$. Then the lower semicontinuity of the perimeter along these sequences guarantees that the infimum in (2.1) is attained.

Now we show that $\mathcal{K}_{q}(\Omega)=\ell_{q}(\Omega)=\min _{\left(E_{1}, E_{2}\right) \in \Phi(\Omega)} Q\left(E_{1}, E_{2}\right)$. Let $\left(\tilde{E}_{1}, \tilde{E}_{2}\right) \in \Phi(\Omega)$ be such that $\ell_{q}(\Omega)=$ $Q\left(\tilde{E}_{1}, \tilde{E}_{2}\right)$. The function $\tilde{u}=\left|\tilde{E}_{2}\right| \chi_{\tilde{E}_{1}}-\left|\tilde{E}_{1}\right| \chi_{\tilde{E}_{2}}$ is an admissible test function in (1.3), and $F_{q}(\tilde{u})=\ell_{q}(\Omega)$. Hence, we have that

$$
\mathcal{K}_{q}(\Omega) \leq \ell_{q}(\Omega) .
$$

In order to conclude the proof, we have to show that, for any admissible function $u \in B V_{0}(\Omega)$,

$$
F_{q}(u) \geq \ell_{q}(\Omega) .
$$


Using standard notation, let $u=u_{+}-u_{-}$, where $u_{+}$and $u_{-}$are, respectively, the positive and negative part of $u$, and set $\Omega_{ \pm}=\operatorname{spt} u_{ \pm}$.

Clearly $\int_{\Omega_{+}} u_{+} \mathrm{d} x=\int_{\Omega_{-}} u_{-} \mathrm{d} x$. Moreover being $u \neq \equiv 0$ implies that $\left|\Omega_{ \pm}\right|>0$. Let

$$
\mu_{+}(t)=\left|\left\{u_{+}>t\right\}\right|, \quad \mu_{-}(t)=\left|\left\{u_{-}>t\right\}\right| .
$$

By the Fubini Theorem and the Hölder inequality, we get that

$$
\begin{aligned}
\int_{\Omega^{+}} u_{+}^{q} \mathrm{~d} x & =\int_{\Omega}\left(u_{+}^{q-1} \int_{0}^{+\infty} \chi_{\left\{u_{+}>t\right\}}(x) \mathrm{d} t\right) \mathrm{d} x \\
& =\int_{0}^{+\infty}\left(\int_{\left\{u_{+}>t\right\}} u_{+}^{q-1} \mathrm{~d} x\right) \mathrm{d} t \leq\left(\int_{\Omega_{+}} u_{+}^{q} \mathrm{~d} x\right)^{1-\frac{1}{q}} \int_{0}^{+\infty} \mu_{+}(t)^{\frac{1}{q}} \mathrm{~d} t
\end{aligned}
$$

(see for example [16], Sect. 1.3.3), and the same relation holds for $u_{-}$. Using the above inequality we deduce that

$$
\left(\int_{\Omega}|u|^{q} \mathrm{~d} x\right)^{\frac{1}{q}} \leq\left[\left(\int_{0}^{\operatorname{ess} \sup u_{+}} \mu_{+}(t)^{\frac{1}{q}} \mathrm{~d} t\right)^{q}+\left(\int_{0}^{\operatorname{ess} \sup u_{-}} \mu_{-}(s)^{\frac{1}{q}} \mathrm{~d} s\right)^{q}\right]^{\frac{1}{q}} .
$$

Now we perform the change of variables

$$
\xi(t)=\int_{0}^{t} \mu_{+}(\sigma) \mathrm{d} \sigma, \quad t \in\left[0, \operatorname{ess} \sup u^{+}\right]
$$

and

$$
\eta(s)=\int_{0}^{s} \mu_{-}(\tau) \mathrm{d} \tau, \quad s \in\left[0, \operatorname{ess} \sup u^{-}\right]
$$

respectively, in both integrals in the right-hand side of (2.2). The functions $\xi$ and $\eta$ are strictly increasing and, being $\int_{\Omega^{+}} u_{+} \mathrm{d} x=\int_{\Omega^{-}} u_{-} \mathrm{d} x:=M$, we have that $\xi(t) \leq M=\xi\left(\operatorname{ess} \sup u^{+}\right)$and $\eta(s) \leq M=\eta\left(\operatorname{ess} \sup u^{-}\right)$. Hence, from (2.2) and the Minkowski inequality (see for example [15], Thm. 202, p. 148) it follows that

$$
\begin{aligned}
\left(\int_{\Omega}|u|^{q} \mathrm{~d} x\right)^{\frac{1}{q}} & \leq\left[\left(\int_{0}^{M} \mu_{+}(t(\xi))^{\frac{1-q}{q}} \mathrm{~d} \xi\right)^{q}+\left(\int_{0}^{M} \mu_{-}(s(\eta))^{\frac{1-q}{q}} \mathrm{~d} \eta\right)^{q}\right]^{\frac{1}{q}} \\
& \leq \int_{0}^{M}\left[\mu_{+}(t(r))^{1-q}+\mu_{-}(s(r))^{1-q}\right]^{\frac{1}{q}} \mathrm{~d} r .
\end{aligned}
$$

On the other hand, denoting by $p_{ \pm}(t)=P\left(\left\{u_{ \pm}>t\right\}\right)$ for $t \geq 0$, the co-area formula for BV functions yields

$$
\begin{aligned}
\|D u\|\left(\mathbb{R}^{n}\right) & =\left\|D u_{+}\right\|\left(\mathbb{R}^{n}\right)+\left\|D u_{-}\right\|\left(\mathbb{R}^{n}\right) \\
& =\int_{0}^{+\infty} p_{+}(t) \mathrm{d} t+\int_{0}^{+\infty} p_{-}(s) \mathrm{d} s=\int_{0}^{M}\left[\frac{p_{+}(t(r))}{\mu_{+}(t(r))}+\frac{p_{-}(s(r))}{\mu_{-}(s(r))}\right] \mathrm{d} r,
\end{aligned}
$$

where we performed the change of variables $t=t(\xi), s=s(\eta)$ defined above. Finally, combining (2.3) and (2.4) we have

$$
\begin{aligned}
F_{q}(u) & \geq \frac{\int_{0}^{M}\left[\frac{p_{+}(t(r))}{\mu_{+}(t(r))}+\frac{p_{-}(s(r))}{\mu_{-}(s(r))}\right] \mathrm{d} r}{\int_{0}^{M}\left[\mu_{+}(t(r))^{1-q}+\mu_{-}(s(r))^{1-q}\right]^{\frac{1}{q}} \mathrm{~d} r} \\
& \geq \inf _{0<r<M} \frac{\frac{p_{+}(t(r))}{\mu_{+}(t(r))}+\frac{p_{-}(s(r))}{\mu_{-}(s(r))}}{\left[\mu_{+}(t(r))^{1-q}+\mu_{-}(s(r))^{1-q}\right]^{\frac{1}{q}}} \geq \inf _{\left(E_{1}, E_{2}\right) \in \Phi(\Omega)} Q\left(E_{1}, E_{2}\right)=\ell_{q}(\Omega),
\end{aligned}
$$

and this concludes the proof. 
Remark 2.1. The Proof of Theorem 1.1 also implies the following property which is similar to the one holding for the standard Cheeger problem (see [19], Rem. 3.2). Assume that for some function $u$ we have $F_{q}(u)=\mathcal{K}_{q}(\Omega)$. Then for almost all ess inf $u<s<0<t<\operatorname{ess} \sup u$ such that the truncated function $v=(u \wedge t) \vee s$ has zero mean value it holds

$$
Q\left(E_{1}, E_{2}\right)=\mathcal{K}_{q}(\Omega) \quad \text { with } E_{1}=\{u>t\} \text { and } E_{2}=\{u<s\},
$$

which to a certain extent corresponds to the idea that almost all super level sets of $u_{+}$and $u_{-}$have to be "optimal".

\section{Proof of Theorem 1.2}

We split the proof into two subsections. In the first one we prove that the minimum of $\mathcal{K}_{q}(\Omega)$ among bounded, open sets of given measure is attained at the union of two disjoint balls. In the second subsection we characterize the minimizers.

\subsection{An isoperimetric inequality for $\mathcal{K}_{q}(\Omega)$}

We denote by $\mathcal{B}(t)$ the family of sets with measure $t$ which are union of two disjoint balls.

Proposition 3.1. Let $\Omega$ be a bounded, open set of $\mathbb{R}^{n}$, with $\Omega \notin \mathcal{B}(|\Omega|)$ and $1 \leq q<1^{*}$. There exists a set $\tilde{\Omega}=\tilde{B}_{1} \cup \tilde{B}_{2} \in \mathcal{B}(|\Omega|)$, such that

$$
\mathcal{K}_{q}(\Omega)>\mathcal{K}_{q}(\tilde{\Omega})=\min _{A \in \mathcal{B}(|\Omega|)} \mathcal{K}_{q}(A)
$$

Moreover

$$
\mathcal{K}_{q}(\tilde{\Omega})=Q\left(\tilde{B}_{1}, \tilde{B}_{2}\right)
$$

Proof. Let $u$ be a minimizer for (1.3). By (1.5) there exists a couple $\left(E_{1}, E_{2}\right) \in \Phi(\Omega)$ such that

$$
\mathcal{K}_{q}(\Omega)=Q\left(E_{1}, E_{2}\right)
$$

The standard isoperimetric inequality implies that

$$
\mathcal{K}_{q}(\Omega)=Q\left(E_{1}, E_{2}\right) \geq Q\left(E_{1}^{\#}, E_{2}^{\#}\right) \geq \min _{\left(F_{1}, F_{2}\right) \in \Phi(A)} Q\left(F_{1}, F_{2}\right)=\mathcal{K}_{q}(A),
$$

where $E_{1}^{\#}, E_{2}^{\#}$ are two disjoint balls such that $\left|E_{i}^{\#}\right|=\left|E_{i}\right|, i=1,2, A=B_{1} \cup B_{2} \in \mathcal{B}(|\Omega|)$, and $E_{i}^{\#} \subseteq B_{i}$, for $i=1,2$. Then there exists $\tilde{\Omega}=\tilde{B}_{1} \cup \tilde{B}_{2} \in \mathcal{B}(|\Omega|)$ such that

$$
\mathcal{K}_{q}(\Omega) \geq \mathcal{K}_{q}(A) \geq \min _{A \in \mathcal{B}(|\Omega|)} \mathcal{K}_{q}(A)=\mathcal{K}_{q}(\tilde{\Omega})
$$

Clearly, the first inequality in (3.3) holds as an equality if and only if $E_{1}, E_{2}$ are balls. In this case, since $\Omega \notin \mathcal{B}(|\Omega|)$, then $\left|E_{1}\right|+\left|E_{2}\right|<|\Omega|$. As matter of fact, it is easy to see that, being $q<1^{*}, Q\left(E_{1}, E_{2}\right)$ is strictly decreasing with respect to homotheties of $E_{1} \cup E_{2}$. This implies that the second inequality in (3.4) is strict, and the proof of (3.1) is completed.

Now suppose that $\mathcal{K}_{q}(\tilde{\Omega})=Q\left(\tilde{E}_{1}, \tilde{E}_{2}\right)$. The couple $\left(\tilde{E}_{1}, \tilde{E}_{2}\right) \in \Phi(\tilde{\Omega})$ is such that each $\tilde{E}_{i}$ is contained just in one ball. Indeed, if for example $\tilde{E}_{1} \cap \tilde{B}_{1}=\tilde{E}_{1}^{a}, \tilde{E}_{1} \cap \tilde{B}_{2}=\tilde{E}_{1}^{b}$, both with positive measure, and $\frac{P\left(\tilde{E}_{1}^{a}\right)}{\left|\tilde{E}_{1}^{a}\right|} \leq \frac{P\left(\tilde{E}_{1}^{b}\right)}{\left|\tilde{E}_{1}^{b}\right|}$, we have that

$$
\frac{P\left(\tilde{E}_{1}\right)}{\left|\tilde{E}_{1}\right|}=\frac{P\left(\tilde{E}_{1}^{a}\right)+P\left(\tilde{E}_{1}^{b}\right)}{\left|\tilde{E}_{1}^{a}\right|+\left|\tilde{E}_{1}^{b}\right|} \geq \frac{P\left(\tilde{E}_{1}^{a}\right)}{\left|\tilde{E}_{1}^{a}\right|},
$$


which implies, being $\left|\tilde{E}_{1}\right|>\max \left\{\left|\tilde{E}_{1}^{a}\right|,\left|\tilde{E}_{1}^{b}\right|\right\}$, that $Q\left(\tilde{E}_{1}, \tilde{E}_{2}\right)>Q\left(\tilde{E}_{1}^{a}, \tilde{E}_{2}\right)$ contradicting the minimality of $\left(\tilde{E}_{1}, \tilde{E}_{2}\right)$. Now suppose that $\tilde{E}_{1} \cup \tilde{E}_{2} \neq \tilde{B}_{1} \cup \tilde{B}_{2}$. Then $\left|\tilde{E}_{1}\right|+\left|\tilde{E}_{2}\right|<|\Omega|$. Moreover, by the standard isoperimetric inequality,

$$
Q\left(\tilde{E}_{1}, \tilde{E}_{2}\right) \geq Q\left(\tilde{E}_{1}^{\#}, \tilde{E}_{2}^{\#}\right)
$$

Finally, being $Q$ strictly monotone with respect to the homotheties, there exist two balls $F_{1}$ and $F_{2}$ such that $\left|F_{1}\right|+\left|F_{2}\right|=|\Omega|$ and

$$
Q\left(\tilde{E}_{1}^{\#}, \tilde{E}_{2}^{\#}\right)>Q\left(F_{1}, F_{2}\right)
$$

contradicting the minimality of $\tilde{\Omega}=\tilde{B}_{1} \cup \tilde{B}_{2}$.

\subsection{Properties of the minimizers and symmetry breaking}

Since the quantity $\mathcal{K}_{q}(\Omega)|\Omega|^{\frac{1}{q}-\frac{n-1}{n}}$ is invariant under dilations, we are free to arbitrarily fix the measure of $\Omega$. Indeed the shape of minimizers of $\mathcal{K}_{q}(\Omega)$ under measure constraint is not affected by the measure chosen.

For simplicity we will restrict our analysis to the case $|\Omega|=\omega_{n}$ (the measure of the unit ball in $\mathbb{R}^{n}$ ). Our minimization problem is

$$
\min _{|\Omega|=\omega_{n}} \mathcal{K}_{q}(\Omega)
$$

and Proposition 3.1 implies that we can reduce ourselves to the study of a one-dimensional minimum problem. More precisely we have to minimize

$$
Q\left(B_{1}, B_{2}\right)=n \omega_{n}^{1-\frac{1}{q}} \frac{r_{1}^{-1}+r_{2}^{-1}}{\left(r_{1}^{-n(q-1)}+r_{2}^{-n(q-1)}\right)^{\frac{1}{q}}},
$$

over all possible pairs of balls $B_{1}$ and $B_{2}$ of radii $r_{1}$ and $r_{2}$ respectively, under the restriction $r_{1}^{n}+r_{2}^{n}=1$.

We introduce the new variable

$$
x=\frac{1}{2} \log \left(\frac{r_{1}^{n}}{1-r_{1}^{n}}\right) .
$$

The value $x=0$ corresponds to $r_{1}=r_{2}$ and $x$ is a monotone increasing function of $r_{1}$ from $-\infty$ to $+\infty$, as $r_{1}$ goes from 0 to 1 . We have,

$$
\min _{|\Omega|=\omega_{n}} \mathcal{K}_{q}(\Omega)=n 2^{\frac{1}{n}} \omega_{n}^{1-\frac{1}{q}} \min _{x \in \mathbb{R}} f_{n}(x, q),
$$

where

$$
f_{n}(x, q)=[\cosh x]^{\frac{1}{n}+\frac{1}{q}-1}\left[\cosh \left(\frac{x}{n}\right)\right][\cosh (x(q-1))]^{-\frac{1}{q}} .
$$

For every $q \in\left[1,1^{*}\left[, f_{n}(0, q)=1\right.\right.$ and obviously $f_{n}(x, q)$ is symmetric about $x=0$. Therefore we also have that $\partial_{x} f_{n}$ vanishes at $x=0$, in fact two balls with equal radii are always a stationary point of the functional $Q\left(B_{1}, B_{2}\right)$. Moreover, $f_{n}(x, q)$ diverges for $|x| \rightarrow \infty$. The behaviour of $f_{n}(\cdot, q)$ is very sensitive to the values of $n$ and $q$ as shown in Figures 1 and 2.

All the statements in Theorem 1.2 are consequences of several claims we are going to prove.

Claim 3.2. For any given $n \geq 2$ and any given $q \in\left[1,1^{*}\left[\right.\right.$, the function $f_{n}(\cdot, q)$ has at most two local minimum points in $[0, \infty[$, and not more than one in $] 0, \infty[$. 


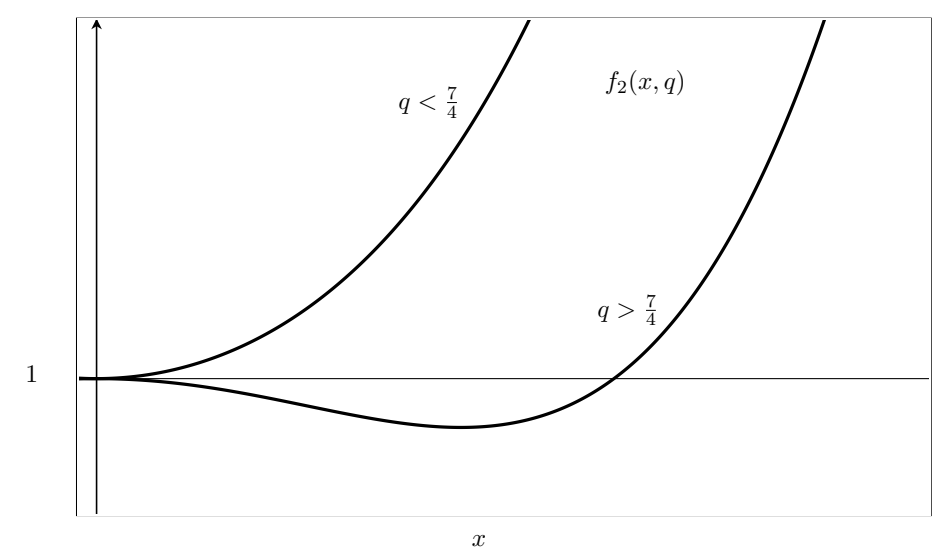

FIGURE 1 . Varying the value of $q$ changes the shape of the function $f_{2}(x, q)$. For $q \leq \frac{7}{4}$ there is only one stationary (global minimum) point in the origin, while for $q>\frac{7}{4}$ another stationary point appears, the origin becomes a local maximum point and the minimum point shifts on positive values.

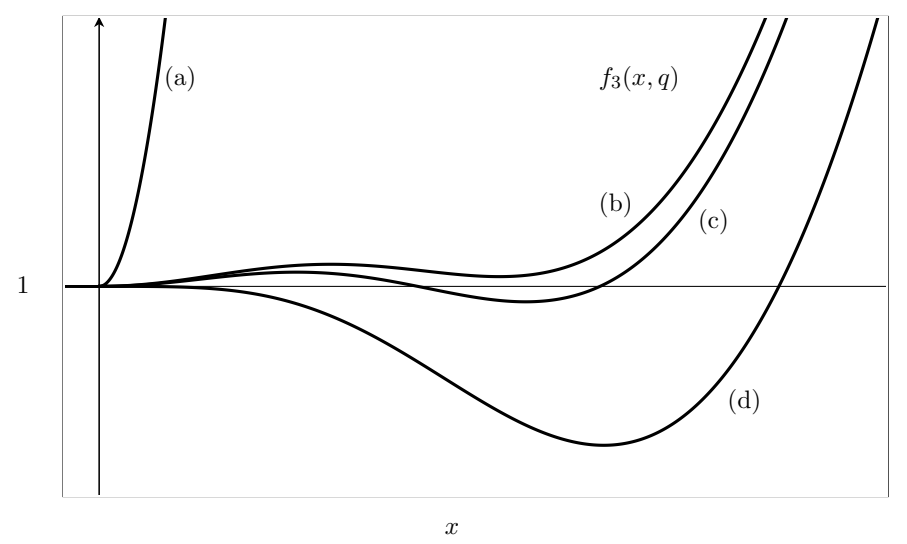

Figure 2. In the picture, we present four different behaviors of the function $f_{3}(x, q)$, corresponding to increasing values of $q$, from (a) to (d). In (a) there is only one stationary point: a global minimum point at 0 . In (b) there are three stationary points and the global minimum point is at 0 . In (c) there are three stationary points and the origin is still a local minimum point yet not a global one. In (d) there are two stationary points and the origin becomes a local maximum point. The picture is about the same for all $n \geq 3$.

Proof of Claim 3.2. Differentiating $f_{n}(x, q)$ with respect to $x$, we have

$$
\begin{aligned}
\partial_{x} f_{n}(x, q)= & 2 c_{n}(x, q)\left\{\left(\frac{1}{n}+\frac{1}{q}-1\right) \cosh \left(\frac{x}{n}\right) \sinh x \cosh (x(q-1))\right. \\
& +\frac{1}{n} \cosh x \sinh \left(\frac{x}{n}\right) \cosh (x(q-1)) \\
& \left.-\left(1-\frac{1}{q}\right) \cosh x \cosh \left(\frac{x}{n}\right) \sinh (x(q-1))\right\}
\end{aligned}
$$


where

$$
c_{n}(x, q)=\frac{1}{2}[\cosh (x(q-1))]^{-\frac{1}{q}-1}[\cosh x]^{\frac{1}{n}+\frac{1}{q}-2}>0 .
$$

By using the well-known identities for $\sinh (a \pm b)$ and $\cosh (a \pm b)$, we get

$$
\begin{aligned}
\partial_{x} f_{n}(x, q)= & c_{n}(x, q)\left\{\left(\frac{1}{n}+\frac{1}{q}-1\right) \sinh \left[x\left(q+\frac{1}{n}\right)\right]+\frac{1}{n} \sinh \left[x\left(2-q+\frac{1}{n}\right)\right]\right. \\
& \left.-\left(1-\frac{1}{q}\right) \sinh \left[x\left(q-\frac{1}{n}\right)\right]\right\}=c_{n}(x, q) A_{n}(x, q),
\end{aligned}
$$

where $A_{n}(x, q)$ is the function in the braces. Since $A_{n}(0, q)=0$, the claim is proved if we show that $A_{n}(\cdot, q)$ has no more than two zeros in $] 0,+\infty[$. This is an immediate consequence of the following.

Lemma 3.3. Any nontrivial linear combination of three hyperbolic sinus functions has at most two positive zeros.

Proof. Let $A(x)=a \sinh (\alpha x)+b \sinh (\beta x)+c \sinh (\gamma x)$, with $a, b$ and $c$ nonzero real numbers, and $\alpha, \beta, \gamma \geq 0$, such that $A \not \equiv 0$. Clearly, if $a, b$ and $c$ have the same sign, the claim of the lemma is obvious. Hence, without loss of generality we can consider linear combinations as follows:

$$
\begin{aligned}
A(x) & =a \sinh (\sqrt{1+\varepsilon} x)+b \sinh (\sqrt{1+\delta} x)-c \sinh x \\
& =X(x)+Y(x)-Z(x)
\end{aligned}
$$

with $a, b, c>0, \varepsilon>-1, \varepsilon \neq 0$ and $\delta \geq-1$. Obviously, $A(0)=0$. Moreover,

$$
A^{\prime \prime}(x)=A(x)+\varepsilon X(x)+\delta Y(x) .
$$

Suppose that there exists a nonpositive local minimum point $x_{0}>0$ of $A$. Then,

$$
A\left(x_{0}\right) \leq 0, \quad A^{\prime \prime}\left(x_{0}\right) \geq 0
$$

and, together with (3.6),

$$
\varepsilon X\left(x_{0}\right)+\delta Y\left(x_{0}\right) \geq 0 .
$$

Moreover, the function $\varepsilon X+\delta Y$ vanishes at 0 , and it cannot have a nonnegative maximum in $] 0,+\infty[$ being, for any $x>0$,

$$
[\varepsilon X(x)+\delta Y(x)]^{\prime \prime}=\varepsilon(1+\varepsilon) X(x)+\delta(1+\delta) Y(x)>\varepsilon X(x)+\delta Y(x) .
$$

Hence,

$$
\varepsilon X(x)+\delta Y(x)>0 \text { for any } x>x_{0} .
$$

Finally, (3.6) and (3.7) imply that

$$
A^{\prime \prime}(x)>A(x) \text { for any } x>x_{0},
$$

and then the function $A(x)$ admits at most one zero in $] x_{0},+\infty[$. This implies also that the function $A$ can vanish at most once in $] 0, x_{0}[$, and the claim follows.

Claim 3.4. For any given $n \geq 2$, and any $x \in] 0, \infty\left[\right.$, the functions $f_{n}(x, \cdot)$ and $\partial_{x} \log f_{n}(x, \cdot)$ are decreasing in $\left[1,1^{*}[\right.$. 
Proof of Claim 3.4. Let us compute

$$
\begin{aligned}
\partial_{q} \log \left(f_{n}(x, q)\right) & =\frac{\partial}{\partial q}\left(\left(\frac{1}{q}+\frac{1}{n}-1\right) \log (\cosh x)-\frac{1}{q} \log (\cosh (x(q-1)))\right. \\
& =-\frac{1}{q^{2}}[\log (\cosh x)-\log (\cosh (x(q-1)))]-\frac{1}{q} x \tanh (x(q-1))<0 .
\end{aligned}
$$

For any fixed $q$, such derivative is decreasing with respect to $x$ in $[0,+\infty[$. Indeed, for $x>0$ we have

$$
\partial_{q x} \log \left(f_{n}(x, q)\right)=-\frac{1}{q^{2}} \tanh x-\frac{1}{q^{2}} \tanh (x(q-1))-x\left(1-\frac{1}{q}\right)[\cosh (x(q-1))]^{-2}<0,
$$

which completes the proof.

Remark 3.5. From the monotonicity of $\partial_{x} \log f_{n}(x, q)$ with respect to $q$ it follows that, if $\log f_{n}(x, \bar{q})$ is increasing with respect to $x$ in $\left[0,+\infty\left[\right.\right.$ for some $\bar{q} \in\left[1,1^{*}[\right.$, then the same holds true for all $q \in[1, \bar{q}[$. Obviously this also means that $f_{n}(x, q)$ is increasing with respect to $x$ for all $q \in[1, \bar{q}$ [ as soon as it is increasing with respect to $x$ when $q=\bar{q}$.

Moreover, since for all $0<x<x_{0}$ and $q \in\left[1,1^{*}[\right.$ the trivial inequality holds

$$
\partial_{q}\left(\log f_{n}(x, q)-\log f_{n}\left(x_{0}, q\right)\right)=-\int_{x}^{x_{0}} \partial_{s q} \log f_{n}(s, q) \mathrm{d} s>0,
$$

we also deduce the following.

Remark 3.6. From Claim 3.4, if $x_{0}>0$ is a global minimum point for the function $f_{n}(\cdot, q)$, for certain $q=q_{0} \in\left[1,1^{*}[\right.$, then for all $q \in] q_{0}, 1^{*}\left[\right.$ global minimum points cannot exist in $\left[0, x_{0}[\right.$. Roughly speaking positive global minimum points of $f_{n}(\cdot, q)$ move left to the right as $q$ increases.

Claim 3.7. If $1 \leq q \leq \frac{7}{4}$, the function $f_{2}(\cdot, q)$ is increasing in $[0, \infty[$.

Proof of Claim 3.7. In view of Claims 3.2 and 3.4 (Rem. 3.5) it is enough to observe that the function $A_{2}\left(\cdot, \frac{7}{4}\right)$ is positive in $] 0, \infty[$. Indeed

$$
\begin{aligned}
A_{2}\left(x, \frac{7}{4}\right) & =\frac{1}{14}\left\{\sinh \left(\frac{9}{4} x\right)+7 \sinh \left(\frac{3}{4} x\right)-6 \sinh \left(\frac{5}{4} x\right)\right\} \\
& =\frac{2}{7} \sinh ^{3}\left(\frac{1}{4} x\right)\left\{6 \cosh x+2 \cosh \left(\frac{3}{2} x\right)-1\right\}>2 \sinh ^{3}\left(\frac{1}{4} x\right)
\end{aligned}
$$

for all $x>0$.

Claim 3.8. If $\frac{7}{4}<q<2$, the function $f_{2}(\cdot, q)$ has a unique local (and global) minimum point $\bar{x}(q)$ in $[0, \infty[$ which is not $x=0$.

Proof of Claim 3.8. We observe that

$$
\partial_{x x} f_{n}(0, q)=c_{n}(0, q) \partial_{x} A_{n}(0, q)=\left(-q+1+\frac{1}{n}+\frac{1}{n^{2}}\right),
$$

hence $\partial_{x x} f_{2}(0, q)<0$ if $q>\frac{7}{4}$. Hence, being $\partial_{x} f_{2}(0, q)=0$, then $x=0$ is a local maximum point for $\frac{7}{4}<q<1^{*}$. From the coercivity of $f_{2}$, a positive minimum point exists and, by Claim 3.2, it is unique.

Remark 3.9. Denoting by $\bar{x}(q)$ the unique minimum point of $f_{2}(\cdot, q), \bar{x}(q):[1,2[\rightarrow[0, \infty[$ is the continuous nondecreasing function represented in Figure 3. 


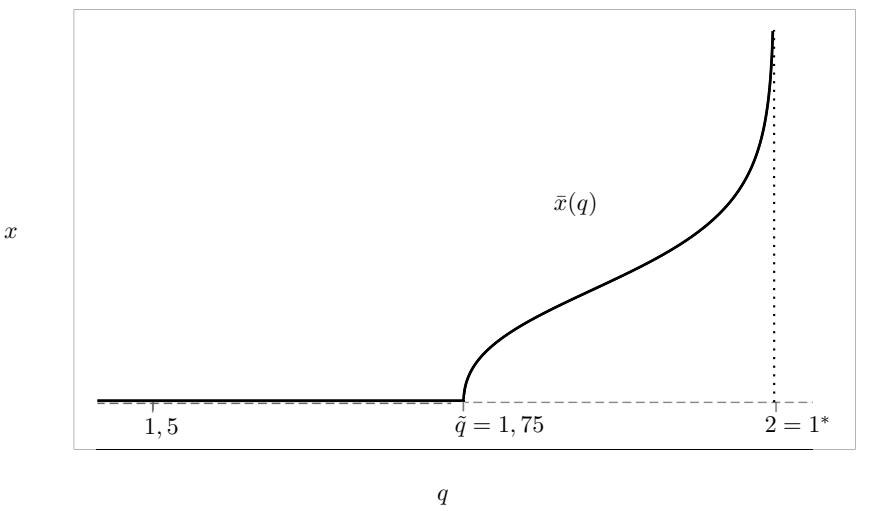

Figure 3. The graph, for $n=2$, of $\bar{x}(q)$ (defined in Claim 3.8). The distance from the origin of the global minimum point is nondecreasing and continuous with respect to $q$.

Claim 3.10. For any $n \geq 3$ and $1 \leq q \leq 1+\frac{1}{n}$, the function $f_{n}(\cdot, q)$ is increasing in $[0, \infty[$.

Proof of Claim 3.10. In view of Claim 3.4 (Rem. 3.5) it is enough to prove the monotonicity in $[0, \infty[$ just for $q=1+\frac{1}{n}$. In fact we have that

$$
f_{n}\left(x, 1+\frac{1}{n}\right)=[\cosh x]^{\frac{1}{n^{2}+n}}\left[\cosh \left(\frac{x}{n}\right)\right]^{\frac{1}{n+1}} .
$$

Claim 3.11. For any $n \geq 3$ and $1+\frac{1}{n}+\frac{1}{n^{2}} \leq q<1^{*}$, the function $f_{n}(\cdot, q)$ has a local maximum point at 0 and a unique local (and global) minimum point in $] 0, \infty[$.

Proof of Claim 3.11. In view of Claim 3.2 and the coercivity of $f_{n}$ with respect to $x$, if $x=0$ is a local maximum point, then $f_{n}$ has a unique local (and global) minimum point in $] 0,+\infty[$. In view of Claim 3.4 (Rem. 3.5) (and the fact that $f_{n}(0, q)=1$ for any admissible $q$ ), it is enough to prove that 0 is a local maximum point for $q=1+\frac{1}{n}+\frac{1}{n^{2}}$. Observe also that for all $1+\frac{1}{n}+\frac{1}{n^{2}}<q<1^{*}$ the statement of Claim 3.11 is a trivial consequence of (3.8). Let us therefore consider $q=\bar{q}=1+\frac{1}{n}+\frac{1}{n^{2}}$. We have that

$$
\partial_{x} f_{n}(x, \bar{q})=c_{n}(x, \bar{q}) A_{n}(x, \bar{q}),
$$

with

$$
\begin{aligned}
A_{n}(x, \bar{q})= & \frac{1}{n\left(n^{2}+n+1\right)}\left\{\sinh \left(\frac{(n+1)^{2}}{n^{2}} x\right)+\left(n^{2}+n+1\right) \sinh \left(\frac{n^{2}-1}{n^{2}} x\right)\right. \\
& \left.-n(n+1) \sinh \left(\frac{n^{2}+1}{n^{2}} x\right)\right\} .
\end{aligned}
$$

A straightforward computation shows that

$$
A_{n}(0, \bar{q})=\partial_{x} A_{n}(0, \bar{q})=\partial_{x x} A_{n}(0, \bar{q})=0,
$$

and

$$
\partial_{x x x} A_{n}(0, \bar{q})=4 \frac{-n^{5}+3 n^{3}+5 n^{2}+4 n+1}{n^{6}\left(n^{2}+n+1\right)} .
$$

For $n=m+3$, the polynomial in the numerator becomes

$$
-m^{5}-15 m^{4}-87 m^{3}-238 m^{2}-290 m-104,
$$




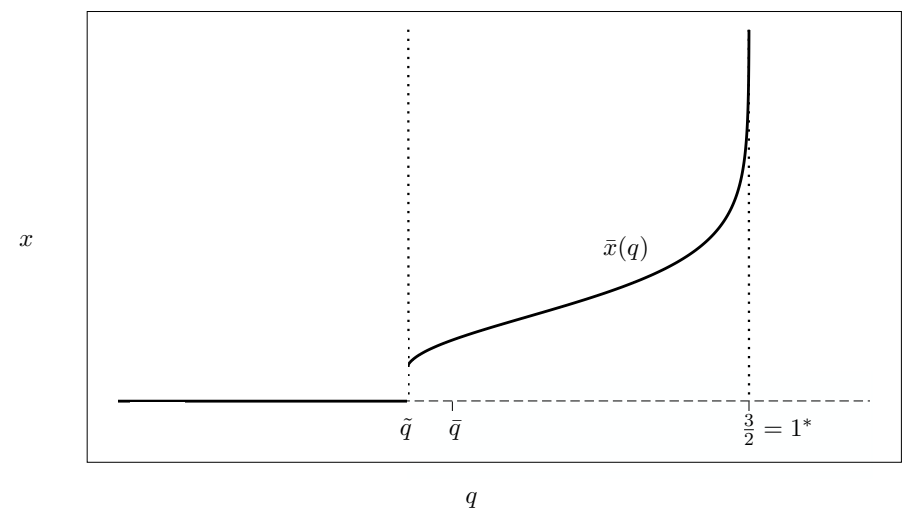

Figure 4. The graph, for $n=3$, of $\bar{x}(q)$ (defined in Claim 3.12). The distance from the origin of the global minimum point is nondecreasing and discontinuous at $q=\tilde{q}$. Here $\bar{q}=\frac{13}{9}$. The behavior is similar for $n>3$ with $\bar{q}=1+\frac{1}{n}+\frac{1}{n^{2}}$.

which is negative if $m=n-3 \geq 0$. Then, in this case we have

$$
\frac{\partial f_{n}}{\partial x}(0, \bar{q})=\frac{\partial^{2} f_{n}}{\partial x^{2}}(0, \bar{q})=\frac{\partial^{3} f_{n}}{\partial x^{3}}(0, \bar{q})=0, \quad \text { and } \quad \frac{\partial^{4} f_{n}}{\partial x^{4}}(0, \bar{q})<0,
$$

which proves that $x=0$ is a local maximum point for $f_{n}(x, \bar{q})$.

Claim 3.12. For any $n \geq 3$, there exists $\tilde{q} \in] 1+\frac{1}{n}, 1+\frac{1}{n}+\frac{1}{n^{2}}\left[\right.$ such that the function $f_{n}(\cdot, \tilde{q})$ has two global minimum points in $\left[0, \infty\left[\right.\right.$, one of which at 0 . As a consequence the function $f_{n}(\cdot, q)$ has a unique global minimum point $\bar{x}(q)$ in $\left[0, \infty\left[\right.\right.$ for all $q \in\left[1,1^{*}\left[-\{\tilde{q}\}\right.\right.$. In particular $\bar{x}(q)=0$ for $q \in\left[1, \tilde{q}\left[\right.\right.$ and $\lim _{q \rightarrow \tilde{q}^{+}} \bar{x}(q)>0$ (see Fig. 4).

Proof of Claim 3.12. We use a continuity argument by taking advantage of the smoothness of $f_{n}$ in $\left[0, \infty\left[\times\left[1,1^{*}[\right.\right.\right.$. All we have to prove is the existence of a value $\tilde{q}$ such that $f_{n}(\cdot, \tilde{q})$ has exactly two global minimum points. Claim 3.2 and the monotonicity properties stated in Claim 3.4 (Rem. 3.5) immediately imply the rest of the statement.

For any given $n \geq 3$, let $\tilde{q}$ be the supremum of all $q \in\left[1,1^{*}\left[\right.\right.$ such that the function $f_{n}(\cdot, q)$ achieves a global minimum at $x=0$. In view of Claim 3.10 and Claim 3.11 we know that $\tilde{q} \in] 1+\frac{1}{n}, 1+\frac{1}{n}+\frac{1}{n^{2}}[$. Since from (3.8) we have $\partial_{x x} f_{n}(0, \tilde{q})>0$, then by definition of $\tilde{q}$ there exists $\tilde{x}>0$ such that $f_{n}(\tilde{x}, \tilde{q})=f_{n}(0, \tilde{q})$. Both $x=0$ and $x=\tilde{x}$ are global minimum points for $f_{n}(\cdot, \tilde{q})$ in $[0, \infty[$ and the claim is proved. With Claim 3.12 the proof of Theorem 1.2 is complete.

Concerning Remark 1.3, the fact that the radius of the largest ball in the minimizers increases with $q$ is a consequence of Claim 3.4 (see Rem. 3.6). Moreover the minimizers degenerate to one ball as $q \rightarrow 1^{*}$ since the minimum point of the function $f_{n}(x, q)$ diverges as $q \rightarrow 1^{*}$. This is a consequence of the fact that $f_{n}(x, q)$ converges as $q \rightarrow 1^{*}$ (monotonically with respect to $q$ ) to

$$
f_{n}^{*}(x)=\left[\cosh \left(\frac{x}{n}\right)\right]\left[\cosh \left(\frac{x}{n-1}\right)\right]^{-1+\frac{1}{n}}
$$

and $f_{n}^{*}(x)$ is in fact decreasing in $[0, \infty[$ in view of

$$
\partial_{x} f_{n}^{*}(x)=-\frac{1}{n}\left[\sinh \left(\frac{x}{n^{2}-n}\right)\right]\left[\cosh \left(\frac{x}{n-1}\right)\right]^{-2+\frac{1}{n}} .
$$




\section{REFERENCES}

[1] L. Barbosa and P. Bérard, Eigenvalue and "Twisted" eigenvalue problems, applications to CMC surfaces. J. Math. Pures Appl. 79 (2000) 427-450.

[2] B. Brandolini, P. Freitas, C. Nitsch and C. Trombetti, Sharp estimates and saturation phenomena for a nonlocal eigenvalue problem. Adv. Math. 228 (2011) 2352-2365.

[3] F. Brock, F. Chiacchio and A. Mercaldo, Weighted isoperimetric inequalities in cones and applications. Nonlinear Anal. 75 (2012) 5737-5755.

[4] A.P. Buslaev, V.A. Kondrat'ev and A.I. Nazarov, On a family of extremal problems and related properties of an integral. Mat. Zametki 64 (1998) 830-838. English transl. Math. Notes 64 (1998) 719-725.

[5] J. Cheeger, A lower bound for the smallest eigenvalue of the Laplacian. Problems in analysis: A symposium in honor of Salomon Bochner. Princeton University Press (1970) 195-199.

[6] A. Cianchi, A sharp trace inequality for functions of bounded variation in the ball. Proc. of Royal Soc. Edinburgh, in vol. 142 . Cambridge University Press (2012) 1179-1191.

[7] G. Croce and B. Dacorogna, On a generalized Wirtinger inequality. Discr. Contin. Dyn. Syst. 9 (2003) 1329-1341.

[8] G. Croce, A. Henrot and G. Pisante, An isoperimetric inequality for a nonlinear eigenvalue problem. Ann. Inst. Henri Poincaré Anal. non Linéaire 29 (2012) 21-34.

[9] B. Dacorogna, W. Gangbo and N. Subía, Sur une généralisation de l'inégalité de Wirtinger. Ann. Inst. Henri Poincaré Anal. Non Linéaire 9 (1992) 29-50.

[10] F. Della Pietra and N. Gavitone, Symmetrization for Neumann anisotropic problems and related questions. Adv. Nonlinear Stud. 12 (2012) 219-235.

[11] F. Della Pietra and N. Gavitone, Relative isoperimetric inequality in the plane: the anisotropic case. J. Convex. Anal. 20 (2013) $157-180$.

[12] L. Esposito, V. Ferone, B. Kawohl, C. Nitsch and C. Trombetti, The longest shortest fence and sharp Poincaré-Sobolev inequalities. Arch. Rational Mech. Anal. 206 (2012) 821-851.

[13] P. Freitas and A. Henrot, On the First Twisted Dirichlet Eigenvalue. Commun. Anal. Geom. 12 (2004) $1083-1103$.

[14] I.V. Gerasimov and A.I. Nazarov, Best constant in a three-parameter Poincaré inequality. Probl. Mat. Anal. 61 (2011) 69-86, (Russian). English transl.: J. Math. Sci. 179 (2011) 80-99.

[15] G.H. Hardy, J.E. Littlewood and G. Pólya, Inequalities. Cambridge University Press, Cambridge (1988).

[16] V.G. Maz'ya, Sobolev spaces with applications to elliptic partial differential equations. Springer, Heidelberg (2011)

[17] A.I. Nazarov, On exact constant in the generalized Poincaré inequality. Probl. Mat. Anal. 24 (2002) 155-180, (Russian). English transl.: J. Math. Sci. 112 (2002) 4029-4047.

[18] A.I. Nazarov, On symmetry and asymmetry in a problem of shape optimization. (2012) 1-5. Available at http://arxiv.org/ abs/1208.3640.

[19] E. Parini, An introduction to the Cheeger problem. Surv. Math. Appl. 6 (2011) 9-21.

[20] E. Parini, The second eigenvalue of the $p$-Laplacian as $p$ goes to 1. Inter. J. Differ. Equ. (2010) D0I:10.1155/2010/984671. 